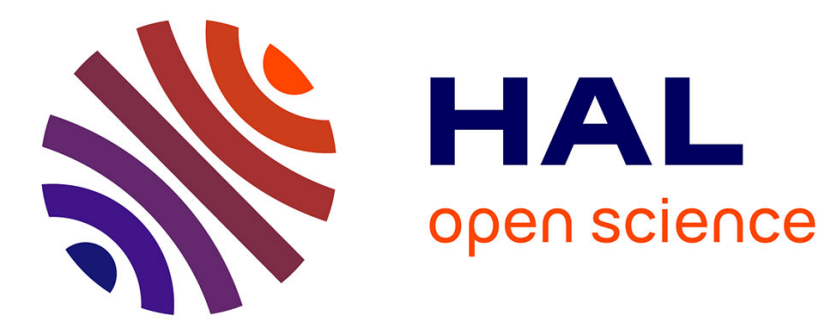

\title{
Les terrasses récentes de la Garonne et leur évolution Jacques Hubschman
}

\section{To cite this version:}

Jacques Hubschman. Les terrasses récentes de la Garonne et leur évolution. Bulletin de l'Association française pour l'étude du quaternaire, 1975, 12 (3-4), pp.137-147. 10.3406/quate.1975.1261 . hal02736172

\section{HAL Id: hal-02736172 \\ https://hal-univ-tlse2.archives-ouvertes.fr/hal-02736172}

Submitted on 2 Jun 2020

HAL is a multi-disciplinary open access archive for the deposit and dissemination of scientific research documents, whether they are published or not. The documents may come from teaching and research institutions in France or abroad, or from public or private research centers.
L'archive ouverte pluridisciplinaire HAL, est destinée au dépôt et à la diffusion de documents scientifiques de niveau recherche, publiés ou non, émanant des établissements d'enseignement et de recherche français ou étrangers, des laboratoires publics ou privés. 


\section{II._- Les terrasses récentes de la Garonne et leur évolution}

\section{Jacques Hubschman}

\section{Abstract}

Description of quaternary alluvial system of the Garonne. The low plain deposits, with brown-leached profiles, are Würm old. The low terrace, with hydromorphic leached soils in silt and already weathered deep horizons in alluvial pebbles, dates from the Riss period.

\section{Citer ce document / Cite this document :}

Hubschman Jacques. II._- Les terrasses récentes de la Garonne et leur évolution. In: Bulletin de l'Association française pour l'étude du quaternaire, vol. 12, n³-4, 1975. pp. 137-147;

doi : https://doi.org/10.3406/quate.1975.1261

https://www.persee.fr/doc/quate_0004-5500_1975_num_12_3_1261

Fichier pdf généré le 19/04/2018 


\section{II. - Les terrasses récentes de la Garonne et leur évolution}

par Jacques HUBSCHMAN

\section{SUMMARY}

Description of quaternary alluvial system of the Garonne. The low plain deposits, with brown-leached profiles, are Würm old. The low terrace, with hydromorphic leached soils in silt and already weathered deep horizons in alluvial pebbles, dates from the Riss period.

\section{I. - LE SYSTEME ALLUVIAL GARONNAIS A L'AVAL DES PETITES PYRENEES}

Au sortir de la cluse prépyrénéenne de BoussensSaint-Martory, la vallée de la Garonne s'épanouit largement dans le bassin molassique, atteignant plus de $25 \mathrm{~km}$ d'est en ouest à la latitude de Toulouse. La dissymétrie qui, à l'ouest de la Garonne, marque les vallées de l'éventail gascon est ici nettement apparente : le fleuve, dominé en rive droite par un raide et haut talus taillé dans la molasse, a laissé sur sa rive gauche un système de terrasses étagées, particulièrement remarquable par son ampleur et sa régularité (fig. 9). C'est du reste surtout entre les latitudes de Muret et de Toulouse que l'escalier de terrasses est à la fois le plus complet et le plus clairement exprimé dans le paysage (fig. 10).

- La nappe culminante de la Garonne que l'on peut attribuer au Donau se suit depuis les Petites Pyrénées jusqu'aux croupes de Lomagne, au nordouest. Disséquée par rivières et vallons, elle revêt aujourd'hui un aspect très discontinu, sous forme de longues lanières légèrement inclinées vers la Garonne, d'échines ou de petits éléments de plateau, eux-mêmes localement étagés. Cette nappe domine la Garonne de plus de $150 \mathrm{~m}$ (près de $200 \mathrm{~m}$ en Lomagne) et vers l'ouest forme balcon au-dessus des coteaux molassiques du Bas-Comminges et de la Gascogne gersoise.

- La haute terrasse, günzienne, est le plus souvent nettement inscrite sous le niveau précédent, à $90 \mathrm{~m}$ environ au-dessus de la Garonne. Elle offre également un aspect dégradé, bien que le plateau de Rieumes et une partie de la Forêt de Bouconne apparaissent encore relativement bien conservés. Ailleurs, et surtout à l'aval de Toulouse, elle ne constitue plus toujours une unité morphologique bien différenciée et se distingue alors malaisément des hauts paliers mindéliens ou du Donau, sinon par ses caractères pédologiques et géochimiques.

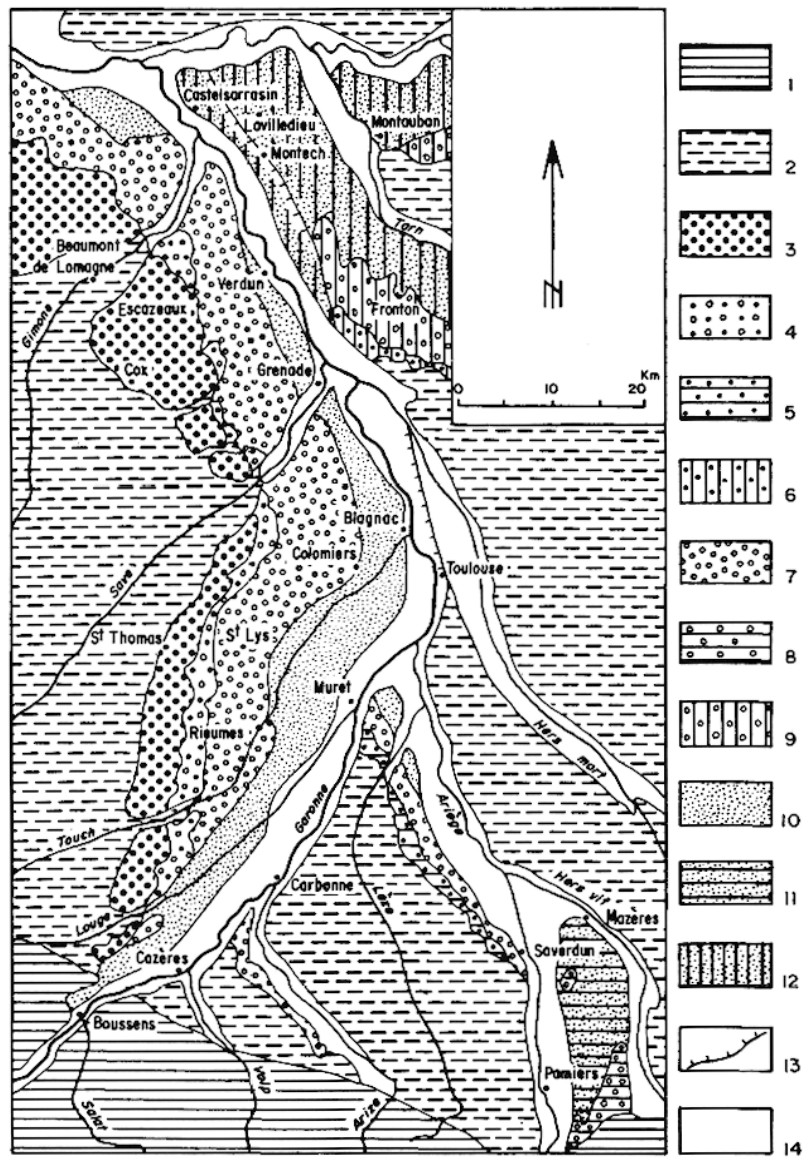

Fig. 9 - Distribution des principales nappes alluviales dans l'avant-pays molassique.

1. Bordure pyrénéenne 2. Molasse 3. Nappe culminante de la Garonne 4. Haute terrasse de la Garonne 5. Lambeaux de la nappe culminante et de la haute terrasse du Tarn 6. Lambeaux de la nappe culminante et de la haute terrasse de l'Ariège 7. Terrasses moyennes de la Garonne et de l'Ariège - rive gauche 8. Haute Boulbonne 9. Terrasse moyenne du Tarn 10. Basscs tcrrasscs de la Garonne et de l'Ariège - rive gauche 11. Basse Boulbonne 12. Plateau de Lavilledieu-Montech et basse terrasse du Tarn 13. Talus limitant le haut palier de la basse plaine garonnaise et le palier inférieur du plateau de Lavilledieu-Montech 14. Basses plaines et lits majeurs. 
- La terrasse moyenne, mindélienne, encore disséquée à proximité des Petites Pyrénées, forme peu à peu vers l'aval l'une des plus remarquables uniţés morphologiques de la région. D'altitude relative variable $(50$ à $80 \mathrm{~m}$ ), elle montre assez fréquemment des paliers discontinus, légèrement dénivellés les uns par rapport aux autres, de 5 à $10 \mathrm{~m}$.

- La basse terrasse rissienne, nettement dominée par le talus de la nappe mindélienne, se poursuit presque sans interruption sur la rive gauche du fleuve depuis la cluse de Boussens jusqu'à la confluence tarnaise. Son altitude relative baisse d'amont vers l'aval, passant de $35 \mathrm{~m}$ à Martres-Tolosane à moins de $20 \mathrm{~m}$, à Verdun-sur-Garonne.

- La basse plaine würmienne est à $25-30 \mathrm{~m}$ à Carbonne mais seulement à moins de $15 \mathrm{~m}$ au-dessus de l'étiage à l'aval de Toulouse. Sous ce niveau s'ìnscrivent les différents faciès du lit majeur postglaciaire et subactuel : ce dernier s'élargit sensiblement dès l'aval immédiat de Toulouse et se distingue alors souvent mal de la basse plaine würmienne proprement dite.

La structure des remblaiements alluviaux offre à peu de choses près les mêmes caractéristiques depuis la nappe culminante danubienne jusqu'à la basse plaine würmienne. Reposant sur la molasse, les alluvions caillouteuses d'épaisseur variable associent galets, graviers et gravillons, emballés dans une matrice sableuse pour les formations récentes, beaucoup plus fine et hétéroclite pour les dépôts anciens altérés. Ces nappes graveleuses sont coiffées en surface par une couverture limoneuse plus ou moins épaisse et caillouteuse, parfois presque entièrement dépourvue d'éléments grossiers (sur l'origine et la mise en place des limons, voir p. 167).

La composition pétrographique des alluvio's caillouteuses trahit l'origine presque exclusivement pyrénéenne du matériel. Les matériaux cristallins, frais ou altérés selon les cas, y dominent de façon générale : granites et quartz surtout mais aussi gneiss et micaschistes, moins fréquemment aplites et pegmatites, beaucoup plus rarement les ophites. Les quartzites sont bien représentés, les grés et éléments schisteux nette- ment moins. Dans les lits majeurs, les alluvions würmiennes et rissiennes, le spectre pétrographique moyen pour les éléments de taille supérieure à $34 \mathrm{~cm}$ est le suivant : 30 à $40 \%$ de matériaux cristallins aluminosilicatés; 20 à $30 \%$ de quartz et fragments de poudingues siliceux; 25 à $35 \%$ de quartzites; 5 à $15 \%$ d'espèces diverses (photo 8). Dans les nappes alluviales antérissiennes, la composition pétrographique initiale apparaît le plus souvent relativement proche de celle des dépôts plus récents, à la différence que, les espèces fragiles ayant été "digérées" ou fortement altérées, les matériaux résistants (quartz, quartzites) prennent le pas dans les spectres pétrographiques. D'autre part, il arrive qu'une certaine évolution se manifeste de l'amont vers l'aval, comme c'est le cas pour la nappe culminante de la Garonne (L. Rieucau, 1958) : les quartz, plus résistants, prennent peu à peu l'avantage sur les autres espèces, notamment les quartzites. Toutefois, ce phénomène est beaucoup moins évident et nc se marque plus très clairement dans les alluvions postérieures au Donau, en particulier dans le Riss et le Würm.

\section{II. - LA BASSE PLAINE WURMIENNE}

La basse plaine a été longtemps considérée comme une formation postglaciaire dans son ensemble (A. Cavaillé 1951, 1965, 1969b), ce que traduisent encore du reste les feuilles au $1 / 50000^{\mathrm{e}}$ de la carte géologique, parues au cours des années 60 . Il est vrai que, comme on l'a vu, à l'aval de Toulouse et plus encore en moyenne Garonne, la basse plaine est occupée par de larges secteurs de dépôts postglaciaires (L. Rieucau, 1971). Cette situation résulte sans aucun doute de la dynamique fluviale propre à cette section du cours d'eau, dynamique qui a brouillé considérablement l'alternance creusement-alluvionnement. Ailleurs en revanche, et particulièrement à l'amont de Toulouse, il est clair que la mise en place du matériel de la basse plaine est nettement antérieure, würmienne. La preuve en est que, par endroit, certains petits gisements loessiques, plaqués au pied du talus dominé

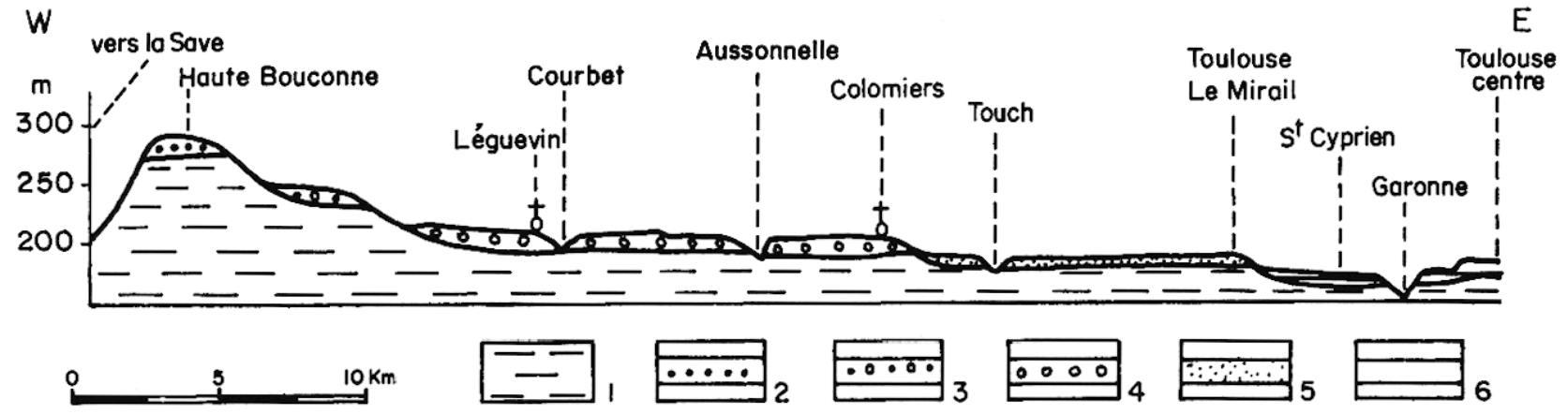

Fig. 10 - Le système alluvial garonnais, au droit de Toulouse. 
$40,23-9,70$

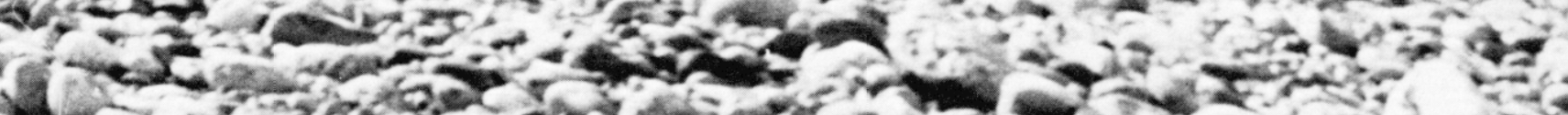

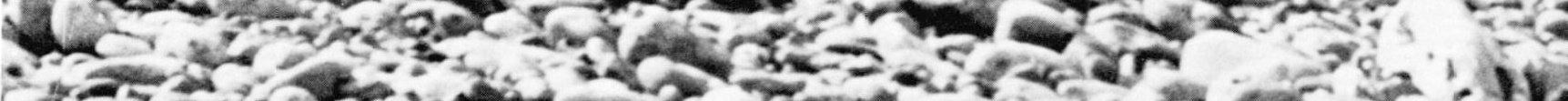

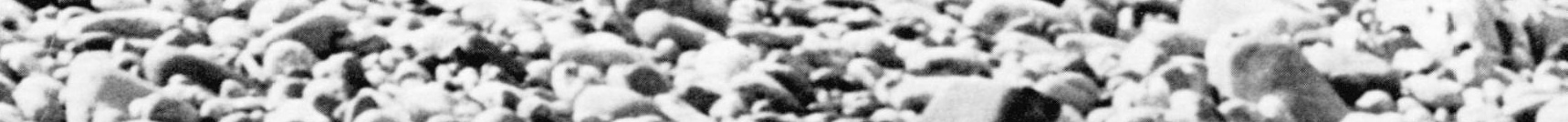

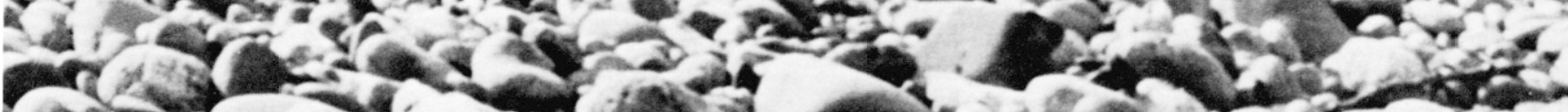

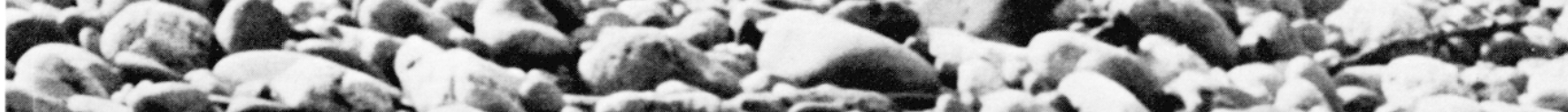

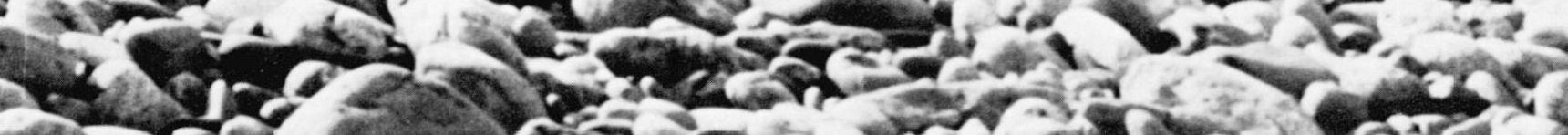

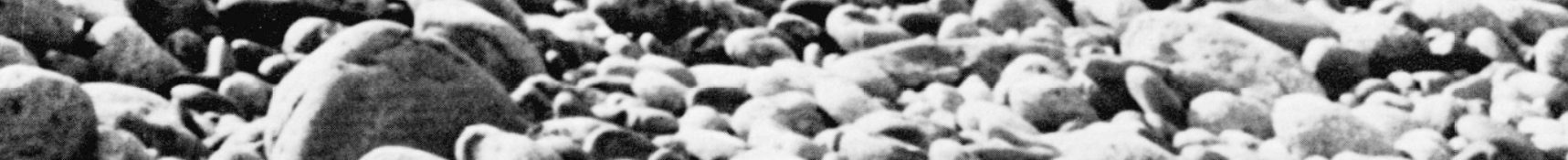

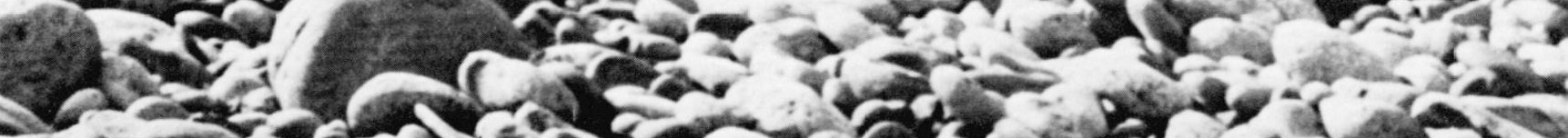

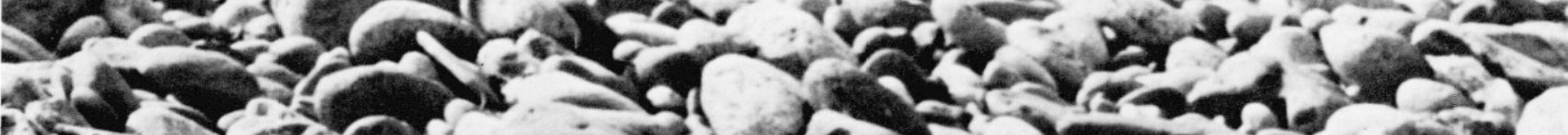

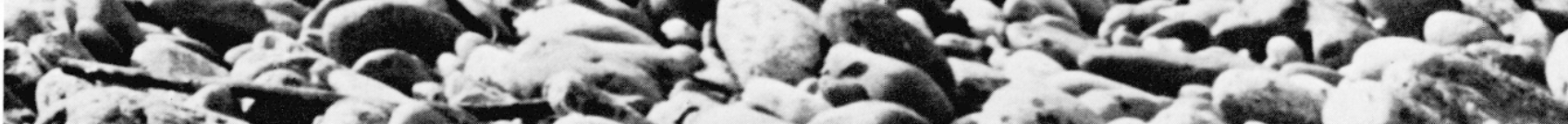

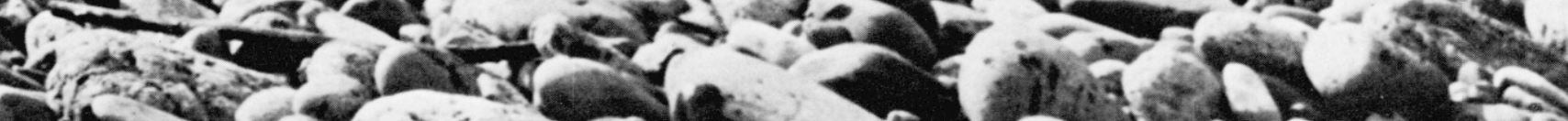

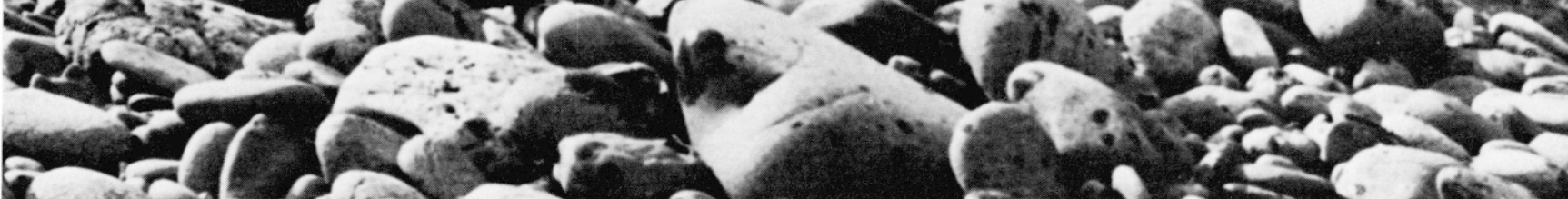
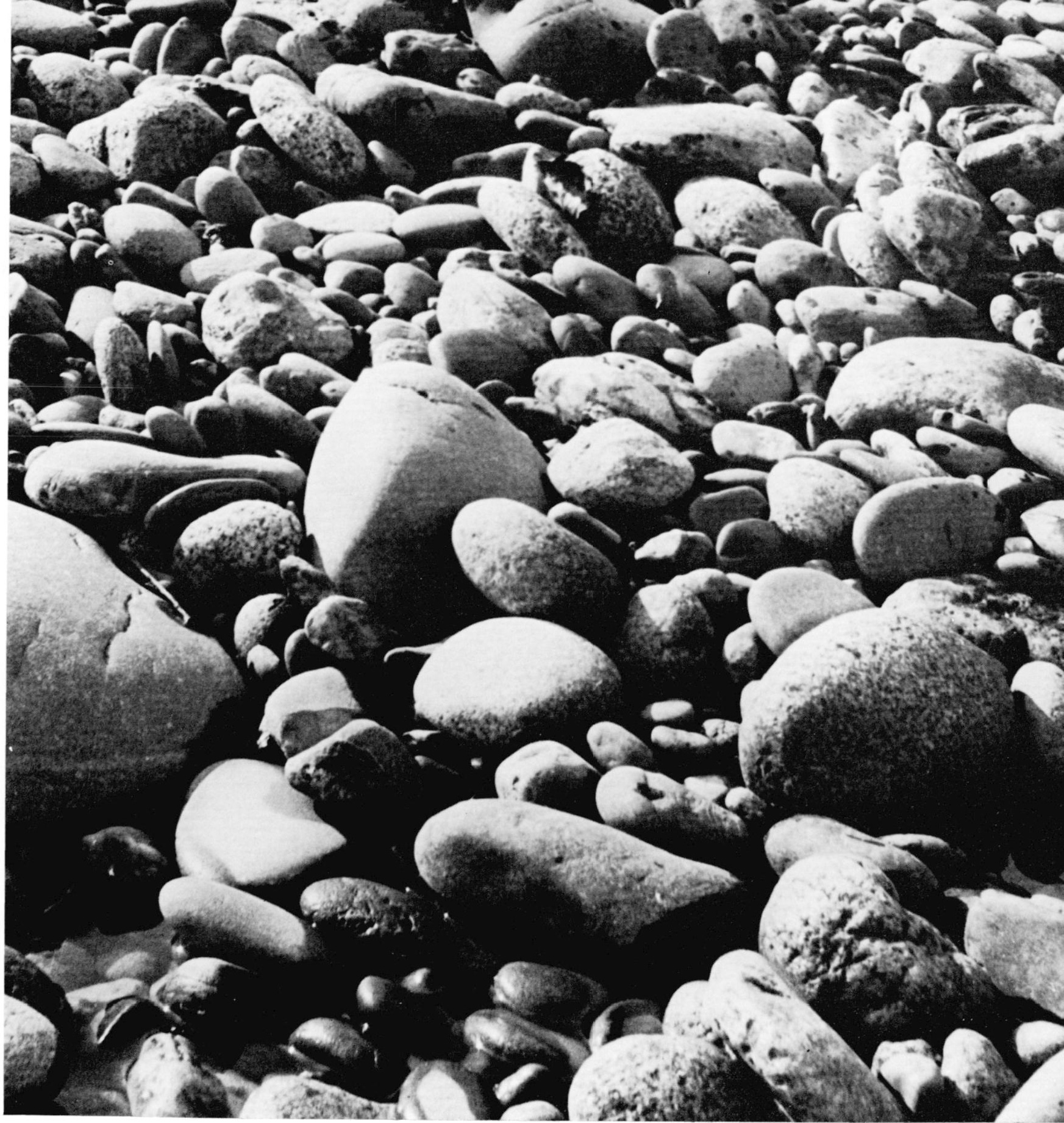

Photo 8 - Pétrographie des cailloutis garonnais (lit majeur) : abondance des quartzites et des éléments gneisso-granitiques. 
par la terrasse rissienne, recouvrent les cailloutis de la basse plaine et passent latéralement aux limons de couverture de cette dernière (J. Hubschman 1975a). Ces dépôts loessiques présentant exactement les mêmes caractères (sédimentologie, exoscopie, concrétionnements, lehm) que la stătion-type de Pompertuzat (p.131), tout indique que l'essentiel du remblaiement de la basse plaine était déjà en place lors du dépôt des loess pendant la seconde moitié du Würm. On serait donc tenté d'attribuer la majeure partie du remblaiement de la basse plaine à un Würm I, correspondant à la phase de progression glaciaire (1). Dans cette optique, le Würm II représenterait la période de dépôt des loess, pendant (ou juste avant) laquelle se seraient étalés les limons alluviaux de couverture, localement éolisés (fig. 3).

D'autre part, alors que les formations épandues au Postglaciaire ne montrent pratiquement aucune évolution pédologique notable, les matériaux würmiens de la basse plaine offrent au contraire des profils clairement différenciés, aussi bien dans les couvertures limoneuses que dans la masse des cailloutis (lorsque l'épaisseur des limons n'est pas excessive). Si le stade des sols lessivés peut être atteint localement, les profils de sols bruns lessivés constituent la règle commune. $\mathrm{La}$ coupe de l'arrêt $\mathrm{n}^{\circ} 6$, trop fraîchement ouverte pour les besoins de l'excursion, encore fort humide et mal exposée, ne donne qu'une image imparfaite de ces sols. La longue carrière de l'arrêt $n^{\circ} 6$ bis, en revanche, bien que très dégradée et peu accessible aujourd'hui (nappe phréatique), permet d'observer le développement général du profil.

(1) Progression glaciaire würmienne dont on étudiera les effets au cours de la $3^{\mathrm{e}}$ journée.

\section{Description sommaire d'un profil de l'arrêt $n^{\circ} 6$ bis}

(partie nord du secteur de carrière, inaccessible aujourd'hui) : sol brun lessivé sur limons et graves :

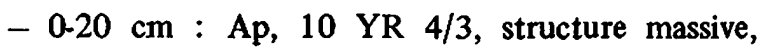
racines, débris divers (carrière), limite nette, ondulée avec $\mathrm{A}_{2}$;

$-20-40 \mathrm{~cm}: A_{2}, 2,5 Y 6 / 2$, structure massive, sous-structure polyédrique localement feuilletée verticalement, graviers et galets TRA* 5 à $15 \%$, limite inférieure graduelle, ondulée ;

- 40-65 cm: $B_{1 t}, 7,5$ YR 6/4 avec revêtements 7,5 YR $5 / 4$ à $4 / 4$, structure polyédrique, revêtements argileux, galets et graviers TRA 10 à $20 \%$, limite inférieure franche, ondulée ;

- 65-120 cm: II $\mathrm{B}_{2 t}$, nappe graveleuse, TRA des galets et graviers 30 à $50 \%$, matrice $5 \mathrm{YR} \mathrm{4/4,}$ structure massive, revêtements argileux, galets patinés et souvent discrètement altérés (cristallins), limite inférieure graduelle ;

- 120-170 cm: II BC à II CB, nappe graveleuse, TRA des galets et graviers 60 à $80 \%$, matrice 5 YR $4 / 4$ à 10 YR 4/4, structure légèrement massive puis particulaire, galets encore assez souvent patinés et discrètement altérés, limite inférieure très graduelle et ondulée (front d'altération) ;

$-170-300 \mathrm{~cm}$ : II C puis II $\mathrm{C}_{\mathrm{g}}$, nappe graveleuse, TRA des galcts et graviers 60 à $80 \%$, matrice 10 YR $6 / 4$, structure particulaire, galets frais et blancs, non altérés. Vers $290 \mathrm{~cm}$, apparition du faciès à pseudogley (bandes horizontales gris-verdâtres, revêtements noirs de fer et manganèse sur les galets), nappe phréatique vers $350 \mathrm{~cm}$.

* TRA : taux de recouvrement apparent.

\begin{tabular}{|c|c|c|c|c|c|c|}
\hline \multirow{3}{*}{$\begin{array}{l}\text { Prof. } \\
\mathrm{cm}\end{array}$} & \multicolumn{6}{|c|}{ Granulométrie \% } \\
\hline & \multirow[b]{2}{*}{ EG } & \multicolumn{5}{|c|}{$\%$ de TF } \\
\hline & & SG & SF & STF & L & A \\
\hline $30-40$ & 43,2 & 23,3 & 18,5 & 18,1 & 20,9 & 17,5 \\
\hline $55-65$ & 75,2 & 33,6 & 12,1 & 9,1 & 16,9 & 27,0 \\
\hline $90-100$ & 84,3 & 53,6 & 8,5 & 4,1 & 8,9 & 23,2 \\
\hline $130-140$ & 81,2 & 71,0 & 10,7 & 2,3 & 5,8 & 9,4 \\
\hline $270-290$ & 79,9 & 86,0 & 3,4 & 1,8 & 4,1 & 4,4 \\
\hline
\end{tabular}

\begin{tabular}{|c|c|c|c|c|c|c|}
\hline \multirow{2}{*}{$\begin{array}{c}\text { Prof. } \\
\text { cm }\end{array}$} & eau & $\mathrm{KCl}$ & $\mathrm{MO}$ & $\mathrm{C}$ & $\mathrm{N}$ & $\mathrm{C}$ \\
\cline { 2 - 7 } & & 4,4 & 1,1 & 0,65 & 0,08 & 7,6 \\
\hline $30-40$ & 5,3 & 4,6 & 0,7 & 0,41 & 0,08 & 5,2 \\
$55-65$ & 5,6 & 4,9 & 0,4 & 0,26 & 0,06 & 4,4 \\
$90-100$ & 5,9 & 5,6 & 0,2 & 0,15 & 0,03 & 5,0 \\
$130-140$ & 6,7 & 5,5 & 0,1 & 0,06 & 0,02 & 2,7 \\
$270-290$ & 6,5 & & & & & \\
\end{tabular}




\begin{tabular}{|c|c|c|c|c|c|c|c|}
\hline \multirow{2}{*}{$\begin{array}{c}\text { Prof. } \\
\text { cm }\end{array}$} & \multicolumn{7}{|c|}{ Complexe absorbant meq \% } \\
\cline { 2 - 7 } & $\mathrm{Ca}$ & $\mathrm{K}$ & $\mathrm{Mg}$ & $\mathrm{Na}$ & $\mathrm{S}$ & $\mathrm{T}$ & $\mathrm{S} / \mathrm{T} \%$ \\
\hline $30-40$ & 3,2 & 0,50 & 0,50 & 0,06 & 4,3 & 8,9 & 48 \\
$55-65$ & 5,8 & 0,25 & 1,1 & 0,06 & 7,3 & 12,2 & 59 \\
$90-100$ & 7,7 & 0,17 & 1,5 & 0,09 & 9,6 & 13,3 & 72 \\
$130-140$ & 3,5 & 0,04 & 0,5 & 0,03 & 4,1 & 7,0 & 59 \\
$270-290$ & 3,0 & 0,04 & 0,5 & 0,03 & 3,6 & 5,3 & 68 \\
\hline
\end{tabular}

\begin{tabular}{|c|c|c|c|}
\hline \multirow{2}{*}{$\begin{array}{c}\text { Prof. } \\
\text { cm }\end{array}$} & FT & FL & FL/FT \% \\
\cline { 2 - 4 } & & 1,06 & 47 \\
$30-40$ & 2,23 & 1,50 & 47 \\
$55-65$ & 3,18 & 1,67 & 49 \\
$90-100$ & 3,35 & 0,71 & 22 \\
$130-140$ & 3,20 & 0,67 & 18 \\
$270-290$ & 3,56 & & \\
\hline
\end{tabular}

\begin{tabular}{|c|c|c|c|c|c|c|c|}
\hline $\begin{array}{c}\text { Prof. } \\
\mathrm{cm}\end{array}$ & Illites & Chlorite & Vermiculite & $\begin{array}{c}\text { Montmo- } \\
\text { rillonites }\end{array}$ & Kaolinites & $\begin{array}{c}\text { Interstr. } \\
10-14 \\
14 \mathrm{C}-14 \mathrm{~V}\end{array}$ & $\begin{array}{c}\text { Interstr. } \\
\text { gonflants }\end{array}$ \\
\hline $30-40$ & $\mathrm{~F}$ & $\mathrm{f}$ & $\mathrm{f}$ & & $\mathrm{f}$ & $\mathrm{m}$ & \\
$55-65$ & $\mathrm{~F}$ & $\mathrm{M}$ & $\mathrm{m}$ & & $\mathrm{f}$ & $\mathrm{f}$ & \\
$90-100$ & $\mathrm{M}$ & $\mathrm{M}$ & $\mathrm{m}$ & & & $\mathrm{f}$ & tr. \\
$130-140$ & $\mathrm{M}$ & $\mathrm{f}$ & $\mathrm{M}$ & & & $\mathrm{m}$ & tr. \\
$270-290$ & $\mathrm{~F}$ & $\mathrm{f}$ & $\mathrm{m}$ & & & $\mathrm{f}$ \\
\hline
\end{tabular}

- Le front d'altération apparaît nettement dans cette coupe. Il correspond essentiellement au passage de l'horizon II BC puis CB à l'horizon II C puis $C_{g}$. De part et d'autre de ce front, en effet, un certain nombre de paramètres changent de caractère :

- le fer, et avant tout le fer "mobile" mis en rapport avec le fer total (FL/FT), est plus abondant au-dessus du front : c'est ce fer qui, patinant les galets et teintant la matrice, visualise en quelque sorte la différenciation entre horizons profonds. Ce phénomène traduit à la fois une certaine illuviation originaire des horizons supérieurs et, surtout, l'altération sur place et l'imprégnation de la matrice, à partir des minéraux primaires inclus dans les sables, les graviers et les galets ;

- les galets cristallins, précisément, ne revêtent pas le même aspect de part et d'autre du front d'altération. En profondeur, dans l'horizon II C, ils sont en majorité frais, blancs, très durs et cohérents (photo 9); ils s'apparentent, en cela, aux faciès observables dans les lits majeurs ou actuels. Au-dessus du front, en revanche, la proportion d'éléments rocheux cristallins discrètement altérés est bien plus élevée (moyenne 10 à $15 \%$ de galets de taille comprise entre 5 et $15 \mathrm{~cm}$, contre moins de $5 \%$ dans les horizons II C). Cette altération se marque soit par une fragilisation générale du galet (feldspaths plus ou moins "brouillés" en lame mince), soit par le caractère mordoré net des biolites, soit par l'apparition des fissures à exsudation rouille ou brune d'oxydes de fer (photo 12);

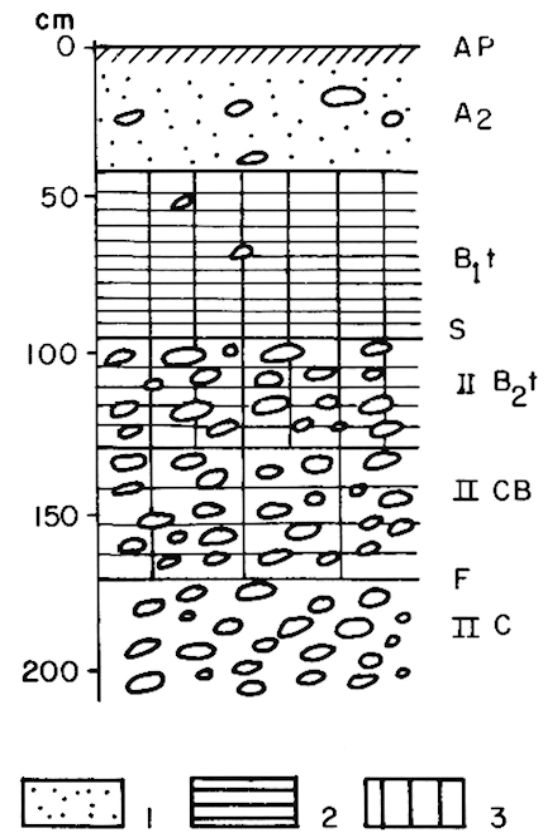

Fig. 11 - Profil schématique des sols bruns lessivés "modaux" des basses plaines de l'avant-pays.

1. Horizon lessivé 2. Accumulation d'argile 3. Accumulation de fer (pour argile et fer, l'espacement des traits est inversement proportionnel à l'importance de l'accumulation) S. Sommet de la nappe graveleuse F. Front d'altération. 


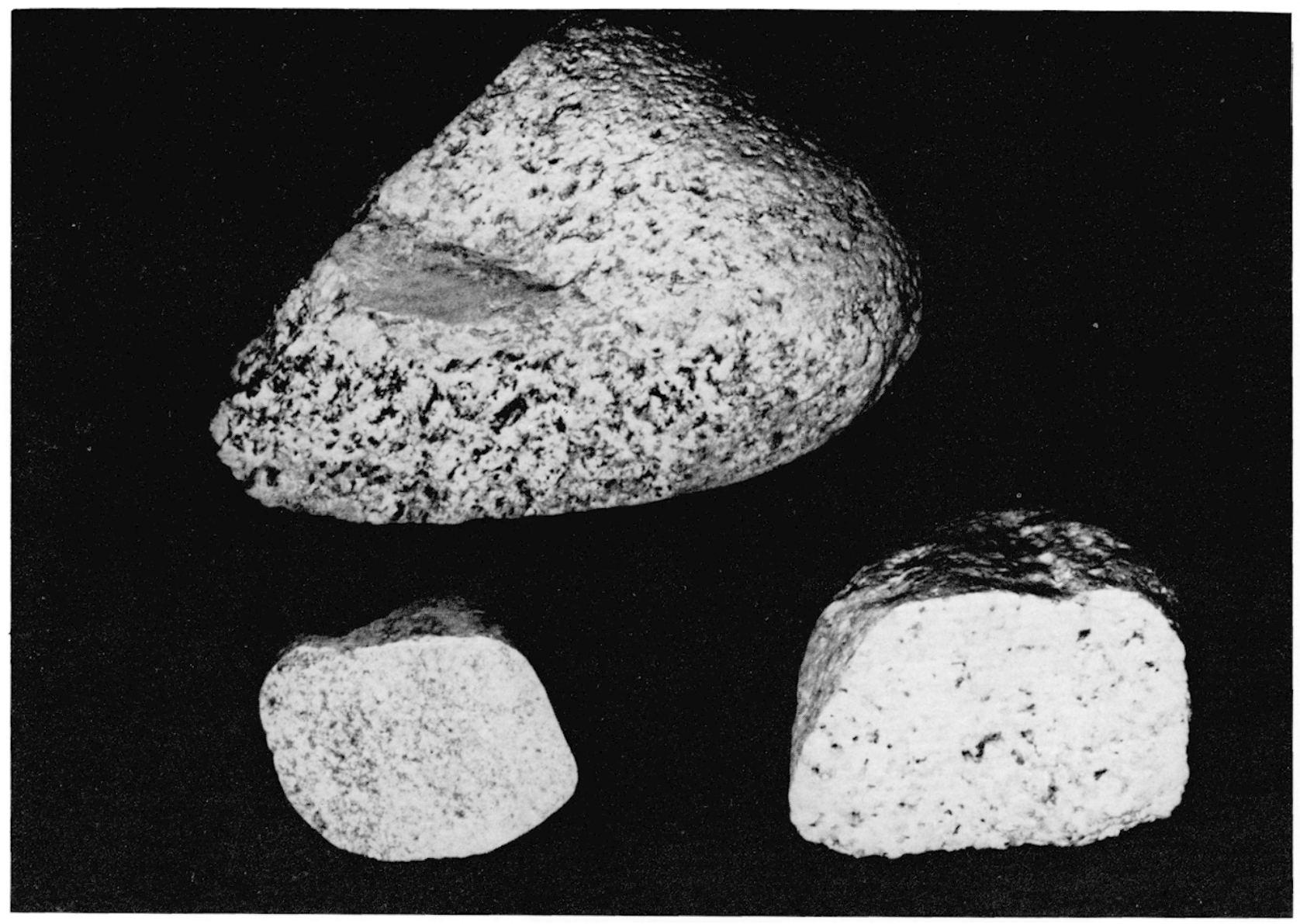

Photo 9 - Terrasse würmienne. Gneiss et granite sains de l'horizon profond II C.

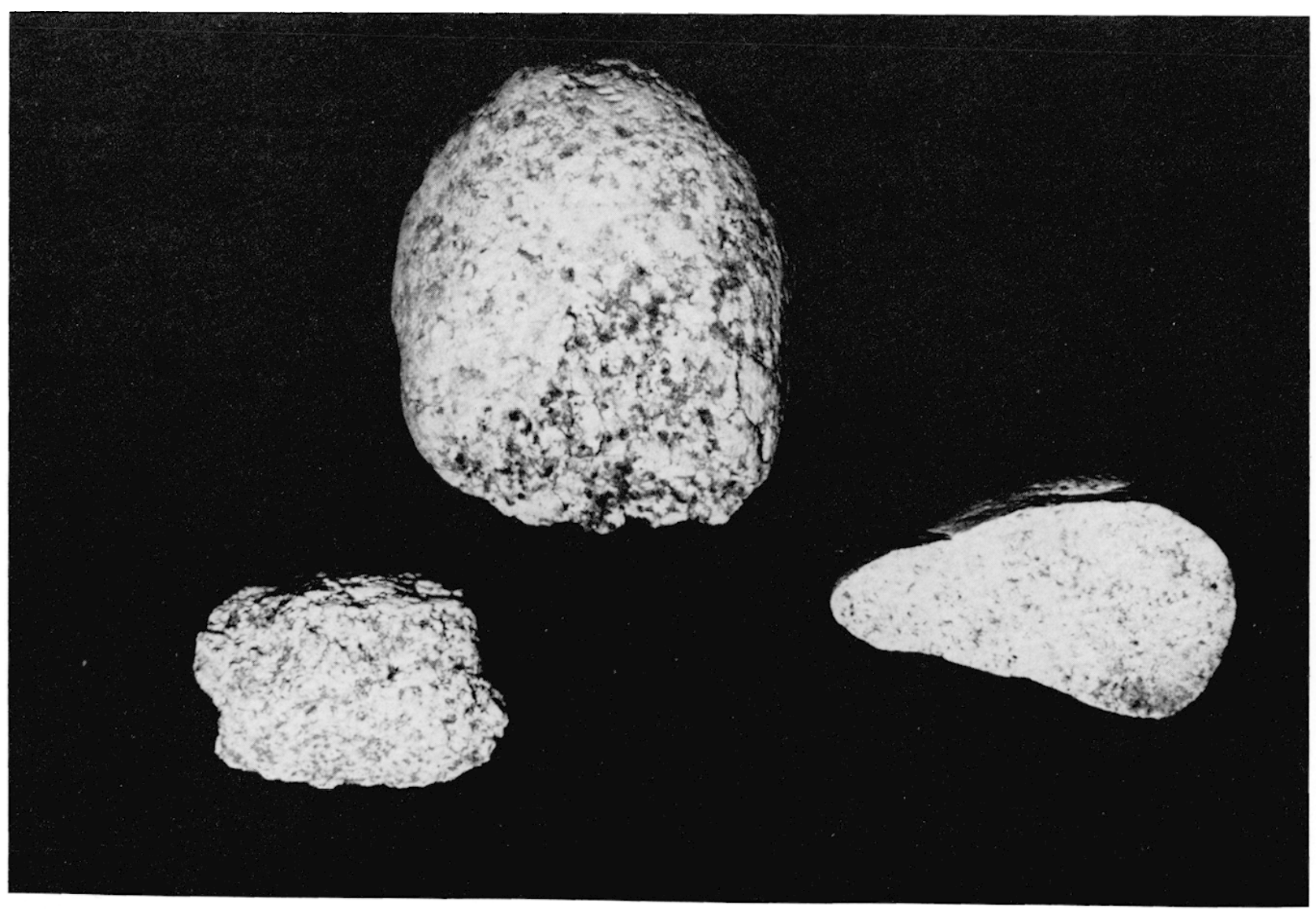

Photo 10 - Terrasse würmienne. Gneiss et granites situés au-dessus du front d'altération. Craquelures et aspect poudreux des feldspaths (au centre et à gauche). 
- les taux d'argiles de la matrice, quoique faibles, sont nettement plus élevés au-dessus du front. La rareté des cutanes d'illuviation indique qu'il s'agit, pour une part assez large, d'une argilisation in situ issue essentiellement de l'altération des aluminosilicates primaires.

- La différenciation texturale et structurale se lit aisément dans les profils. Sous des $A_{2}$ de teinte terne, de structure massive à tendance légèrement feuilletée, les horizons argilliques, bien structurés et à cutanes bien exprimés, montrent une couleur plus vive, encore mieux marquée dans les horizons II $B_{t}$ développés dans le sommet de la nappe caillouteuse. Dans son ensemble, il s'agit donc d'un profil déjà clairement différencié à tous égards (fig. 11).

- L'évolution des minéraux argileux le long du profil n'appelle pas de commentaires particuliers. On notera simplement que les structures gonflantes sont pratiquement inexistantes (sauf dans l'horizon II $\mathrm{C}_{\mathrm{g}}$ - donnée non publiée ici-). On notera également l'importance des illites (plus abondantes en surface) et la relative faiblesse des structures interstratifiées 10-14 et $14 \mathrm{C}-14 \mathrm{~V}$ : ces dernières, associées aux minéraux de type vermiculite et chlorite-Al (ou secondaire), vont prendre, au contraire, un grand développement dans les horizons limoneux des nappes rissiennes.

\section{III. - L'EVOLUTION DE LA BASSE TERRASSE RISSIENNE}

De la même façon que la basse plaine a été pendant longtemps considérée comme postglaciaire dans son ensemble, on a attribué à la basse terrasse garonnaise un âge globalement würmien (A. Cavaillé 1965, 1969b et feuilles locales de la carte géologique au $1 / 50000^{\mathrm{e}}$ ). Cette datation reposait essentiellement sur l'outillage, de faciès moustérien, récolté sur les basses terrasses aussi bien garonnaises que tarnaises. A elles seules, pourtant, l'évolution des profils et le degré d'altération de la basse terrasse, nettement plus poussés que dans la basse plaine, contredit singulièrement cette attribution (J. Hubschman 1972b). Une preuve supplémentaire - et sans doute, définitive - de l'âge rissien de la basse terrasse vient d'être apportée par A. Tavoso (1975) qui a mis en évidence d'importantes stations de bifaces acheuléens dans les basses terrasses du confluent TarnAgout.

Alors que la basse plaine würmienne porte le plus souvent des profils de sols bruns lessivés, développés surtout dans la couverture limoneuse, l'évolution pédologique de la basse terrasse rissienne se caractérise généralement par des sols lessivés hydromorphes. Les coupes étudiées lors de l'arrêt $\mathrm{n}^{\circ} 7$, à proximité du talus dominant la basse plaine würmienne, sont très significatives à cet égard et représentent assez bien le type de différenciation et d'altération le plus couramment observé dans la basse terrasse. Manquent cependant ici les horizons caillouteux très profonds et la zone du front d'altération, qui sont visibles à l'arrêt 8 (Toulouse, sous le Géant-Casino). Pour des raisons de commodité, on décrit ci-dessous une coupe profonde et complète, peu éloignée de l'arrêt 7 (à proximité de Plaisance-du-Touch) et dont les paramètres tant morphologiques qu'analytiques ne diffèrent pas outre mesure.

\section{Description sommaire d'un profil voisin de l'arrêt $n^{\circ} 7$ \\ sol lessivé hydromorphe}

- 0-15 cm: Ap, 10 YR 6/2, structure massive, sous-structure granulaire puis lamellaire, racines, galeries, graviers TRA 1 à $3 \%$, limite inférieure graduelle, ondulée ;

$-15-30 \mathrm{~cm}: A_{21}, 10 \mathrm{YR} 7 / 3$, structure massive, sous-structure discrètement lamellaire puis à tendance polyédrique, pores et canalicules, racines, gravillons, limite graduelle, ondulée ;

$-30-60 \mathrm{~cm}: A_{22}$ puis $B_{11 \mathrm{tg}}, 10 \mathrm{YR} 6 / 3$ avec traînées 10 YR $5 / 4$ en profondeur, structure légèrement lamellaire puis polyédrique, fissures, revê. tements argileux, points noirs ou rouille, graviers et galets TRA 2 à $3 \%$, limite graduelle, ondulée ;

$-60-75 \mathrm{~cm}: B_{12 \mathrm{tg}}$, idem avec polyèdres et pseudogley mieux exprimés, limite graduelle, ondulée ;

$-75-95 \mathrm{~cm}: \mathrm{B}_{2 \mathrm{tg}}, 10 \mathrm{YR} 5 / 4$ avec panachage vertical $10 \mathrm{YR} 4 / 4$ et $10 \mathrm{YR} 6 / 3$, structure polyédrique, revêtements argileux, points noirs et ocres, quelques revêtements noirs, graviers et gravillons TRA $5 \%$, limite inférieure assez franche, ondulée, avec concrétions rondes noires et revêtements noirs sur galets ;

- 95-120 cm: II $B_{3 t g}$, nappe graveleuse de graviers et galets, TRA 60 à $70 \%, 40$ à $60 \%$ des galets cristallins très fragilisés, rubéfiés ou arénisés, ensemble patiné, matrice 7,5 YR 4/4 à 5 YR 4/4, structure massive à tendance polyédrique, revêtements argileux, enduits et concrétions noirs de fermanganèse surtout au sommet, limite inférieure graduelle, ondulée ;

$-120-230 \mathrm{~cm}$ : II $\mathrm{BC}_{\mathrm{g}}$, idem avec peu de revê. tements argileux, moins de concrétions et enduits noirs, matrice 5 YR 4/4 à 10 YR 6/4, structure massive, limite inférieure graduelle, ondulée ;

- $230-400 \mathrm{~cm}$ : II CB, idem avec absence de faciès pseudogley et de revêtements argileux, 10 à $20 \%$ seulement de galets cristallins altérés, matrice 10 YR $6 / 4$ à 2,5 Y 5/4, structure massive à particulaire, limite inférieure graduelle, ondulée ;

- $400-470 \mathrm{~cm}$ : II C, nappe graveleuse relativement fraîche, galets non ou peu patinés, matrice $2,5 \mathrm{Y} 6 / 2$, structure particulaire. 


\begin{tabular}{|c|c|c|c|c|c|c|}
\hline \multirow{3}{*}{$\begin{array}{l}\text { Prof. } \\
\mathrm{cm}\end{array}$} & \multicolumn{6}{|c|}{ Granulométrie \% } \\
\hline & \multirow[b]{2}{*}{ EG } & \multicolumn{5}{|c|}{$\%$ de $\mathrm{TF}$} \\
\hline & & SG & SF & STF & $\mathrm{L}$ & A \\
\hline $15-30$ & 5,6 & 9,8 & 22,8 & 30,5 & 16,7 & 18,5 \\
\hline $40-50$ & 0 & 9,7 & 19,3 & 31,9 & 17,0 & 20,8 \\
\hline $60-75$ & 0 & 6,9 & 19,4 & 29,0 & 17,7 & 25,4 \\
\hline $80-90$ & 0 & 5,9 & 19,3 & 26,6 & 15,7 & 31,1 \\
\hline $100-110$ & 30,2 & 23,1 & 8,9 & 12,1 & 12,8 & 41,6 \\
\hline $190-200$ & 54,8 & 58,7 & 9,9 & 3,0 & 5,1 & 21,3 \\
\hline $330-340$ & 44,1 & 69,1 & 9,7 & 2,0 & 4,9 & 14,1 \\
\hline $450-460$ & 69,9 & 81,6 & 7,4 & 1,2 & 3,0 & 6,3 \\
\hline
\end{tabular}

\begin{tabular}{|c|c|c|c|c|c|c|c|c|}
\hline \multirow{2}{*}{$\begin{array}{c}\text { Prof. } \\
\mathrm{cm}\end{array}$} & \multicolumn{2}{|c|}{$\mathrm{pH}$} & \multicolumn{2}{|c|}{$\mathrm{P}_{2} \mathrm{O}_{5} \% 0$} & \multicolumn{4}{|c|}{ Matière organique $\%$} \\
\hline & eau & $\mathrm{KCl}$ & citrique & Joret & MO & $\mathrm{C}$ & $\mathrm{N}$ & $\mathrm{C} / \mathrm{N}$ \\
\hline $15-30$ & 6,2 & 5,4 & 0,27 & 0,11 & 1,0 & 0,63 & 0,08 & 7,5 \\
\hline $40-50$ & 6,3 & 5,4 & 0,28 & 0,11 & 0,6 & 0,39 & 0,06 & 6,7 \\
\hline $60-75$ & 6,5 & 4,8 & 0,39 & 0,17 & 0,5 & 0,33 & 0.05 & 5,8 \\
\hline $80-90$ & 6,2 & 5,0 & 0,35 & 0,16 & 0,5 & 0,30 & 0,06 & 5,2 \\
\hline $100-110$ & 6,0 & 5,0 & 0,18 & 0,09 & 0,8 & 0,49 & 0,06 & 7,8 \\
\hline $190-200$ & 6,1 & 5,0 & - & - & - & - & - & - \\
\hline $330-340$ & 6,0 & 5,1 & - & - & - & - & - & - \\
\hline $450-460$ & 6,1 & 5,2 & - & - & - & - & - & - \\
\hline
\end{tabular}

\begin{tabular}{|c|r|r|r|r|r|r|r|}
\hline \multirow{2}{*}{$\begin{array}{c}\text { Prof. } \\
\mathrm{cm}\end{array}$} & \multicolumn{7}{|c|}{ Complexe absorbant meq \% } \\
\cline { 2 - 8 } & $\mathrm{Ca}$ & $\mathrm{K}$ & $\mathrm{Mg}$ & $\mathrm{Na}$ & $\mathrm{S}$ & $\mathrm{T}$ & $\mathrm{S} / \mathrm{T} \%$ \\
\hline $15-30$ & 6,5 & 0,12 & 1,7 & 0,06 & 8,4 & 10,3 & 81 \\
$40-50$ & 5,7 & 0,12 & 1,8 & 0,06 & 7,8 & 9,4 & 82 \\
$60-75$ & 6,4 & 0,17 & 2,8 & 0,06 & 9,5 & 11,8 & 80 \\
$80-90$ & 7,6 & 0,23 & 3,7 & 0,09 & 11,6 & 14,8 & 78 \\
$100-110$ & 10,7 & 0,36 & 5,3 & 0,19 & 16,6 & 19,6 & 84 \\
$190-200$ & 5,0 & 0,60 & 2,4 & 0,09 & 8,1 & 10,1 & 8,0 \\
$330-340$ & 4,3 & 0,21 & 2,0 & 0,05 & 6,5 & 9,3 & 7,0 \\
$450-460$ & 4,0 & 0,25 & 1,6 & 0,05 & 5,9 & 8,9 & 6,7 \\
\hline
\end{tabular}

\begin{tabular}{|c|c|c|c|}
\hline \multirow{2}{*}{$\begin{array}{c}\text { Prof. } \\
\text { cm }\end{array}$} & FT & Fe \% & FL/FT \% \\
\cline { 2 - 4 } & & & 41 \\
\hline $15-30$ & 1,67 & 0,70 & 41 \\
$40-50$ & 1,89 & 0,78 & 41 \\
$80-75$ & 2,12 & 0,89 & 40 \\
$100-110$ & 2,73 & 1,11 & 35 \\
$140-150$ & 4,07 & 1,84 & 39 \\
$190-200$ & 3,02 & 1,19 & 23 \\
$430-340$ & 3,21 & 0,05 & 19 \\
\hline
\end{tabular}

\begin{tabular}{|c|c|c|c|c|c|}
\hline $\begin{array}{c}\text { Prof. } \\
\mathrm{cm}\end{array}$ & Illites & $\begin{array}{c}\text { Chlorite } \\
\text { primaire }\end{array}$ & C-V-Al* & Kaolinites & $\begin{array}{c}\text { Interstr. } \\
\text { gonflants }\end{array}$ \\
\hline $15-30$ & $\mathrm{~F}$ & $\mathrm{f}$ & $\mathrm{M}$ & & \\
$40-50$ & $\mathrm{M}$ & $\mathrm{tr}$ & $\mathrm{F}$ & $\mathrm{f}$ & \\
$60-75$ & $\mathrm{~F}$ & $\mathrm{f}$ & $\mathrm{M}$ & $\mathrm{f}$ \\
$80-90$ & $\mathrm{M}$ & $\mathrm{f}$ & $\mathrm{M}$ & $\mathrm{m}$ \\
$100-110$ & $\mathrm{~F}$ & $\mathrm{f}$ & $\mathrm{m}$ & $\mathrm{m}$ \\
$190-200$ & $\mathrm{M}$ & $\mathrm{m}$ & $\mathrm{m}$ & $\mathrm{m}$ & $\mathrm{tr}$ \\
$330-340$ & $\mathrm{M}$ & $\mathrm{m}$ & $\mathrm{m}$ & & \\
\hline $50-460$ & $\mathrm{M}$ & $\mathrm{m}$ & & & \\
\hline
\end{tabular}

* C-V-Al : minéraux à comportement de vermiculite ou chlorite-Al, ou d'interstratifiés 10-14 C, 10-14 V, 14 C-14 V. 


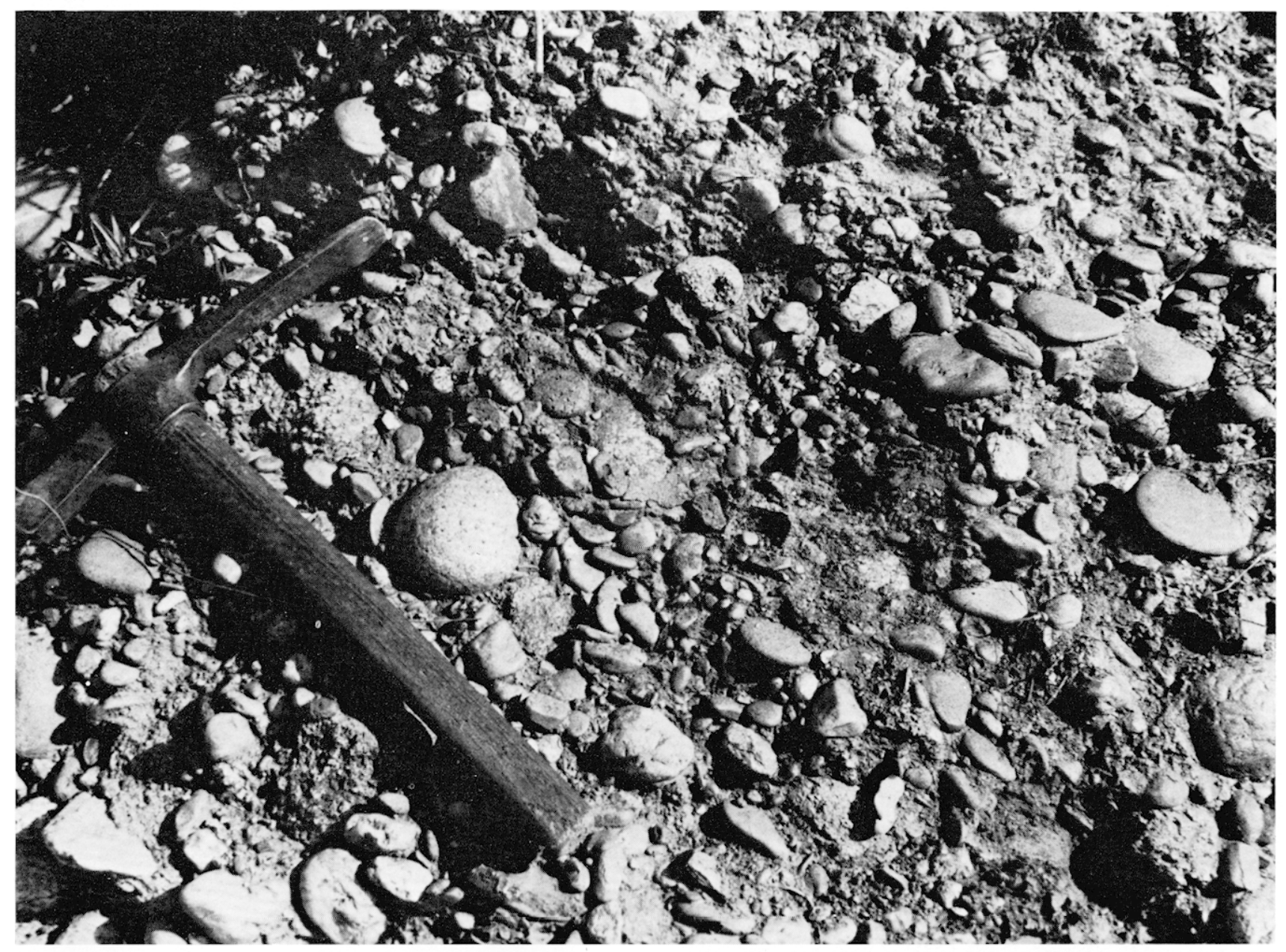

Photo 11 - Terrasse rissienne. Altération du cailloutis : de part et d'autre du manche de piochon, cristallins altérés bien visibles.

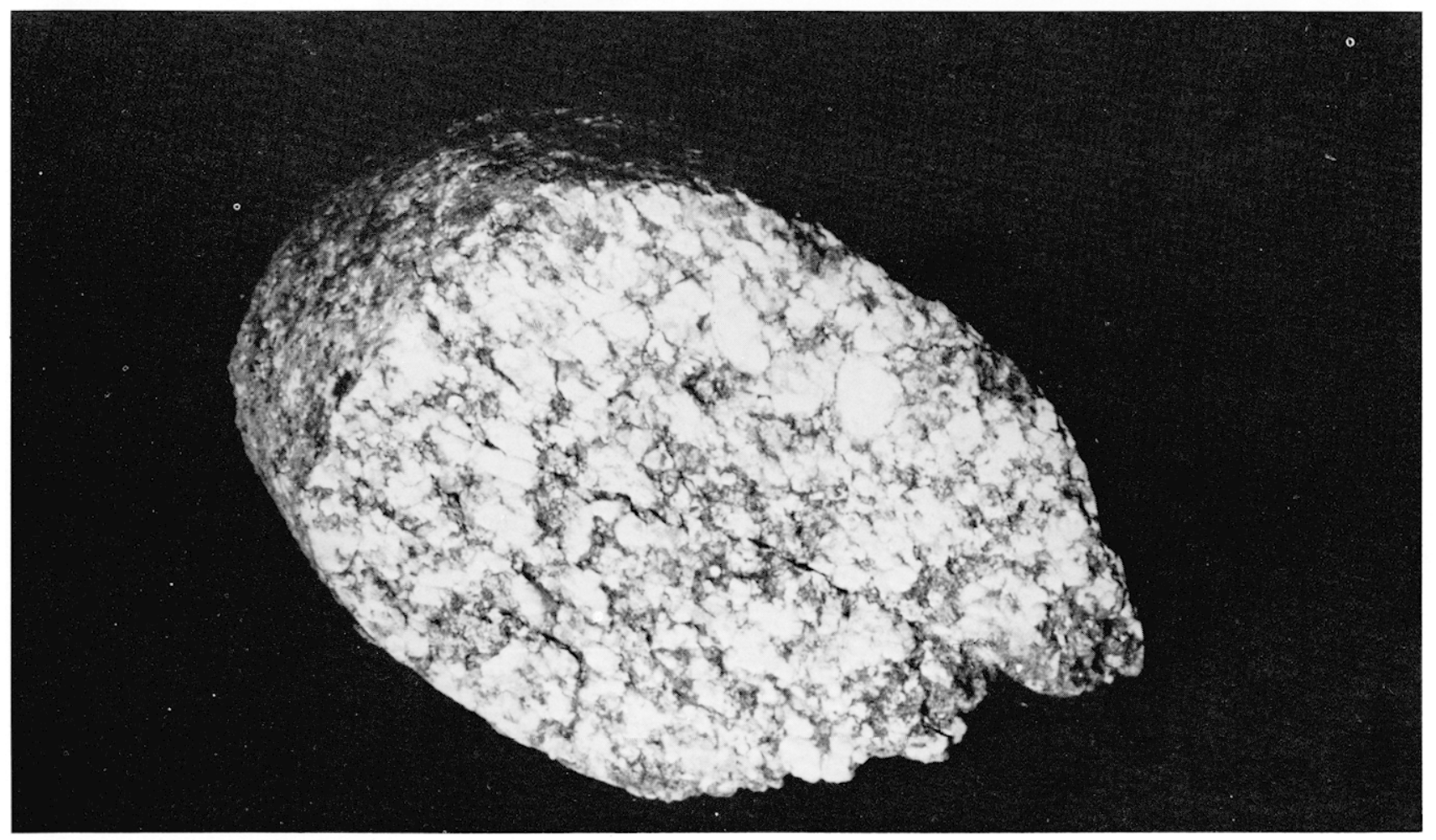

Photo 12 - Terrasse rissienne. Gneiss en voie d'arénisation (en coupe) : feldspaths plus ou moins poudreux et légère argilisation interstitielle. 
- Le front d'altération, dont la profondeur oscillait entre 150 et $200 \mathrm{~cm}$ dans la basse plaine würmienne, atteint généralement 4 mètres (et dépasse souvent cette valeur) dans la basse terrasse rissienne, comme le montre l'arrêt $n^{\delta} 8$. Sous le front d'altération, on retrouve un horizon graveleux II $\mathrm{C}$ relativement sain : c'est la "grave blanche" qu'utilisent à l'occasion les entreprises de construction lorsque la profondeur des fondations atteint ou dépasse 4 mètres environ. La majeure partie des galets cristallins reste non (ou très peu) altérée, la matrice est claire et pauvre en fer libre, les taux d'argiles sont le plus souvent compris entre 5 et $10 \%$ (1). Au-dessus de ce front d'altération, en revanche, les horizons II $\mathrm{BC}$ et II $\mathrm{CB}$ diffèrent très nettement de leurs homologues développés dans la basse plaine würmienne.

- L'altération affecte généralement ici la moitié au moins (et souvent beaucoup plus) des galets cristallins non exclusivement quartzeux. Ces derniers sont en voie d'arénisation plus ou moins avancée : fendillements avec exsudation du fer, plagioclases poudreux, galets presque entièrement pulvérisés (photos 11 et 12). Les autres éléments cristallins aluminosilicatés, apparemment indemnes, sont, en réalité, bien souvent discrètement touchés (friabilité au marteau, craquelures épigénisées par les oxydes et hydroxydes de fer...).

. La matrice qui emballe la grave est nettement plus colorée que dans les profils würmiens, en liaison avec des taux de libération du fer plus élevés : à la limite, et si l'on prend et compte les horizons II $B_{t}$, ces profils caillouteux discrètement rubéfiés pourraient être qualifiés de "faiblement fersiallitiques".

- La différenciation texturale et l'argilisation contrastent aussi singulièrement avec celles des basses plaines würmiennes. D'abord, les profils développés dans la couverture limoneuse ressortissent ici aux sols lessivés hydromorphes. L'épais horizon II $B_{t}$ qui prolonge, au sommet des graves, les $B_{t}$ limoneux, offre un très net faciès argillique (abondants cutanes et revêtements dans les cavités matricielles des galets), qui s'estompe progressivement vers le bas. Ensuite, la matrice des horizons II BC et II CB, au-dessus du front d'altération, est bien plus riche en particules fines que dans les profils würmiens. Comme dans ces derniers, il est probable que non seulement l'illuviation, mais également l'argilogénèse in situ, sont responsables de ces teneurs en particules argileuses.

(1) Il n'est pas rare que l'horizon II C soit le siège de phénomènes d'hydromorphie relativement marqués, liés à l'existence d'une nappe phréatique bloquée au toit de la molasse. Dans ce cas, l'altération est beaucoup plus poussée, au point que le front d'altération ne se distingue plus clairement dans un profil aux caractères "brouillés" par l'hydromorphie et la redistribution du fer qu'elle provoque.

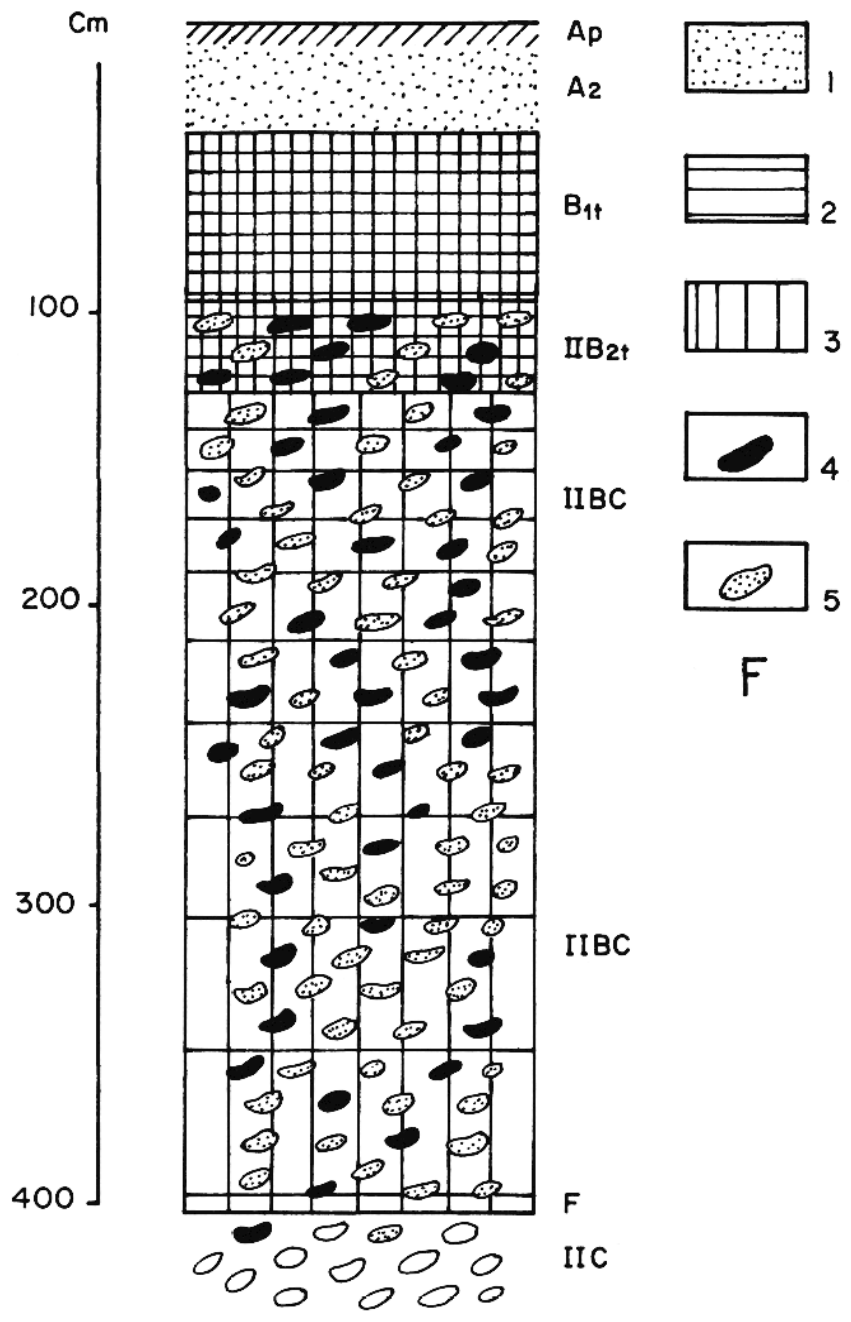

Fig. 12 - Profil schématique des sols lessivés des basses terrasses de l'avant-pays molassique et des niveaux homologues. 1. Horizon lessivé 2. Accumulation d'argile 3. Accumulation de fer (pour argile et fer, l'espacement des traits est inversement proportionnel à l'importance de l'accumulation) 4. Galets de roche cristalline en voie d'arénisation plus ou moins marquée 5. Galets patinés F. Front d'altération.

On note la profondeur du front d'altération, de l'ordre de 4 à $5 \mathrm{~m}$, et un certain gradient d'altération des galets cristallins avec la profondeur.

- L'hydromorphie affecte déjà de façon sensible la plupart des profils rissiens. Cette hydromorphie est, en règle générale, surtout secondaire et induite par la formation des horizons argilliques, eux-mêmes marqués par la redistribution du fer. A cet égard, le contact limon/cailloutis constitue très fréquemment la zone privilégiée pour l'individualisation du fer (et du manganèse qui l'accompagne habituellement), sous forme de concrétions rondes, de revêtements, d'enduits et d'épigénisation locales des graviers et galets. Par endroit, cette individualisation se renforce et entraîne le développement d'un véritable horizon concrétionné par le fer (grep) : ce faciès, qui n'est pas rare dans la basse terrasse rissienne, est cependant beaucoup plus répandu dans les nappes alluviales antérieures. 
- L'évolution des minéraux argileux, comparée à celle de la basse plaine würmienne, se caractérise surtout par le développement massif, dans les couvertures limoneuses, de minéraux à comportement de vermiculites ou d'interstratifiés $14 \mathrm{C}-14 \mathrm{~V}$, plus rarement 10-14 C ou 10-14 V. Cet ensemble de structures phylliteuses a été réuni en une seule rubrique, dont la majeure partie est assimilable en première analyse aux chlorites secondaires et vermiculites-Al. Leurs propriétés sont aujourd'hui bien connues (Y. Tardy, 1969) et ces minéraux abondent dans les limons franchement acides et lessivés, au détriment des illites et surtout des chlorites primaires. S'ils sont encore très bien représentés dans les horizons argilliques Il $B_{t}$, au sommet des cailloutis, ils s'estompent assez rapidement en profondeur. On notera également l'apparition de structures gonflantes, peu abondantes, dans les horizons les plus affectés par l'hydromorphie, et la rareté des minéraux kaoliniques.

- L'organisation générale des profils rissiens, enfin, est marquée par un gradient vertical d'altération clairement exprimé. Sous les horizons différenciés dans la couverture limoneuse, en effet, les alluvions graveleuses offrent des faciès qui évoluent peu à peu avec la profondeur. Du haut vers le bas du profil, des horizons II $B_{t}$ aux horizons II $\mathrm{C}$, la succession des faciès pédologiques et géochimiques traduit une double séquence :

- une séquence progressive d'appauvrissement de la matrice, par estompage de l'illuviation argileuse et ferrugineuse, et décroissance de l'argilisation et de l'imprégnation ferrugineuse issues de l'altération in situ;

. une séquence progressive d'enrichissement de la grave en éléments cristallins peu ou pas altérés, qui évolue en sens inverse de la précédente (fig. 12). Ce modèle d'organisation verticale, qui était déjà en germe dans la basse plaine würmienne, se retrouvera de façon très générale dans les nappes alluviales antérissiennes et en particulier dans les formations mindéliennes et günziennes. Mais, d'un étage stratigraphique à l'autre, ce modèle va évoluer et présenter des paramètres spécifiques à chaque génération de remblaiement alluvial.

\section{Bibliographie}

(Voir p. 214). 